in the lens prevent a clear view except at an extreme angle, an adjustment which cannot be obtained for a camera. Detachments of the retina with bands of proliferation in the vitreous are at present outside the scope of photography. The representation of such conditions has to be a composite picture, so as to show the several planes. The human eye and hand at every turn have to make allowances and ignore opacities which in photography would give little else than a blurred negative, and the exposures necessary for focussing varying depths would put a severe tax upon the steadiness and endurance of the patient. Conceivably some means might be devised for capturing the inverted image, but as photography of this class would demand intelligent co-operation, not as a rule an outstanding virtue among hospital patients, the results would be disappointing.

These remarks, partaking of arguments which might be resorted to by an advocatus diaboli, are not intended to disparage the labours of others in photographing the fundus, but merely to indicate problems which must be solved before an accurate and instructive print can be obtained.

NotE.-Since the above was written I have had the opportunity of seeing a number of drawings by that veteran ophthalmologist, Mr. Pridgin Teale, happily still with us. They were made in the year 1866, on the minute scale adopted about that time. No exception can be taken to his faithful records of detachment, opaque nerve fibres, and rupture of the choroid. They are far in advance of many published by his earlier contemporaries.

\title{
THREE CASES OF GAS INFECTION OF THE CORNEA, FOLLOWING GUNSHOT WOUNDS OF THE EYE
}

\author{
BY \\ J. A. Pringle, M.D., \\ CAPTAIN, R.A.M.C.
}

DURING the present war gas gangrene has been so frequent a complication of gunshot wounds of other parts of the body that ophthalmic surgeons working at military hospitals must long ere this have been struck by the extreme rarity of the condition as aftecting the eye. So far I do not know of any case having been recorded ; in fact, I can discover no record of gas gangrene having occurred in face injuries of any sort. This immunity is probably due to two main factors - the extremely good blood supply of these parts, and the fact that dirty clothing is not carried into the wounds with the fragments of metal, with the added factor as far as the eye 
alone is concerned that the conjunctival sac is not a favourable situation for the development of anaerobic organisms. 'The first of these factors does not apply to the cornea, however, as this structure is non-vascular, and entirely dependent on the lymph flow for its nutrition; any serious interference with its supply of lymph causing great damage to its vitality. In war injuries to the eye this lowering of the vitality of the cornea occurs in a considerable number of cases, but not necessarily as a result of a direct injury to the cornea; it also occurs when the ocular conjunctiva is extensively damaged, with resulting congestion and chemosis, the swollen conjunctiva very often completely hiding the cornea for the first few days. If, therefore, the organisms of gas gangrene are present either in the conjunctival sac or in the. wound, the conditions necessary for their growth and development could not be more favourable.

It was not until the spring of 1917 that a case occurred in my wards, which, by its unusual features, led me to speculate as to the possibility of the cornea being the seat of a gas infection. This case unfortunately was not investigated bacteriologically, and except for the fact that my two subsequent cases followed an almost identical course, I have no grounds for recording it in this article. I merely mention it here as being the first case which aroused my suspicions and put me on the look-out for similar cases.

In June of the same year I was able for the first time to confirm my suspicions by bacteriological investigation. The patient, a private in the Army Ordnance Corps, aged 25, was admitted on June 17, with wounds of the face and eyes due to detonator explosion three days previously. His face was covered with multiple superficial wounds, all of which were septic and discharging pus. My notes on his eye condition were:

Right eye.-Vision=P.L. Superficial wounds of inner side of cornea with some dirt in the wounds. None appears to have perforated. Eye congested and dirty with muco-purulent discharge from conjunctival sac. Fundus and media normal. Left eye.-Vision $=$ No P.L. Wound of cornea inner edge-perforating-edges of wound infiltrated. Eye very inflamed; mucopurulent discharge.

These notes were made when I saw the case in the morning about 9.30 o'clock. I saw the patient again about 2.30 p.m., and by that time the whole left cornea had become infiltrated, soft, and swollen, and was bulging forward at the wound; the eye was intensely inflamed and the ocular conjunctiva chemosed.

I removed the eye that afternoon, leaving a collar of sclera round the nerve head, and thoroughly irrigating the socket with 1-5,000 sublimate. The patient made an uninterrupted recovery, and was transferred to England on June 22 with a perfectly clean socket and $6 / 18$ vision in the remaining eye. Portions of the cornea and 
contents of the globe were retained for bacteriological examination, and Captain Riddle, R.A.M.C., kindly furnished me with a short report, stating that he had isolated staphylococci and a gas-forming bacillus. Unfortunately, Captain Riddle was posted to another unit before completing his investigations, and he was therefore unable to give me a fuller report on the case.

I was now convinced in my own mind that gas infection of the cornea did occur, but owing to the incomplete report of the bacteriologist, I determined to wait' until a third case came under my observation, in order to obtain a full bacteriological investigation. The opportunity did not arise until about nine months later, although many hundreds of patients passed through the eye wards during this period. As this third case presents one or two striking features, demonstrating macroscopically, as it did, the presence of gas-forming organisms in the cornea, and as I am able to submit a very complete bacteriological report from Major S. W. Patterson, R.A.M.C., it may be of interest if I record my observations in detail.

Pte. H. L., of the South Wales Borderers, aged 19, was admitted to the eye wards from convoy on May 1, 1918, having been wounded two days previously. The notes accompanying him on his field medical card from the Casualty Clearing Station, and dated the day he was wounded, were: Small wound of entry inner canthus right eye, globe apparently uninjured, haemorrhage nose and mouth, foreign body removed from in front of left tonsil; dry pack; stitch left in tongue. His condition on arrival was: Temperature $102^{\circ} \mathrm{F}$., pulse 108 , wound of entry at right inner canthus; packed; much swelling of right lids and right side of face and neck. Great chemosis of lower conjunctiva. Loss of inner half of lower lid and small portion of upper. Lower and inner third of cornea uncovered except by chemosed conjunctiva. Cornea bright, slight proptosis, and some limitation of movements of globe down and in. Tension of globe good. Vision = P.L. Some blood-stained discharge from right nostril. Mouth rather foul. The packing was removed from the wound, which was septic and foul smelling, and the track gently syringed through into the mouth with warm saline.

On May 2 the wound in the mouth had closed, and the entrance wound at the inner canthus was much cleaner. The temperature was still $102^{\circ} \mathrm{F}$., and pulse 104 , but his condition was good. I asked Captain Harty, the nose and throat specialist, to see the case, and he very kindly examined it for me. He reported a very longstanding ethmoiditis with hypertrophic rhinitis, and recommended nasal douching with saline and the use of collosol argentum afterwards.

By May 6 the patient's temperature had come down to normal and pulse to 80 , and any danger of meningitis owing to a fracture 
through the cribriform plate of the ethmoid was now at an end. The wound at the inner canthus was discharging freely, and, in spite of frequent bathings, the right eye was continually fouled by the discharge. The chemosis of the conjunctiva had, if anything, increased, and was now hiding the inner and lower half of the cornea, which had become slightly hazy. By means of adhesive strapping, the lower lid was held up in such a position as to cover the cornea. Atropin drops were ordered once a day, with 15 per cent. argyrol drops night and morning, along with frequent bathings with warm boric lotion.

Two days later an ulcer of the cornea developed over the lower and inner part, but there was very little infiltration of the corneal substance, and the ulcer looked remarkably clean and superficial.

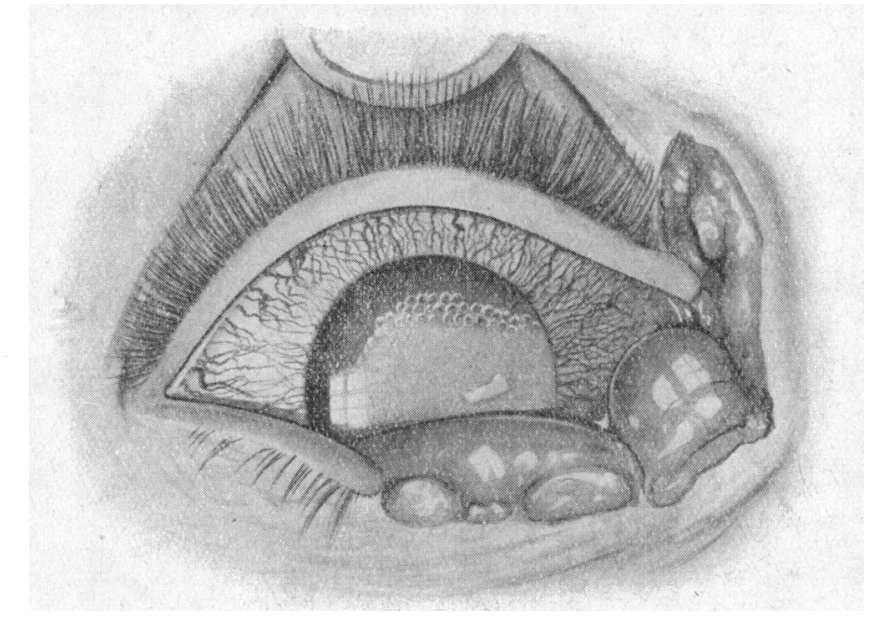

10.45

Next day the eye looked rather better and the ulcer had not increased in size, but when I saw the case about 10.45 o'clock on the following morning the whole appearance had changed. The upper outer conjunctiva, which up to this had shown only mild inflammatory reaction, was now intensely congested and slightly chemosed. The lower and inner two-thirds of the cornea had become completely opaque, looked swollen and oedematous, and was beginning to bulge forward at the site of the ulcer. Along the upper and outer edge of the infiltrated area a double row of minute bubbles of gas could be distinctly seen with the naked eye. These bubbles appeared to be in the superficial layers of the true cornea, and presented a very striking picture as they were hidden only by a slight haze of the intervening substance, while the cornea beyond them was almost clear. Half an hour later the bubbles had increased 
in size and were spreading towards the upper and outer edge of the cornea, the infiltration spreading up with them and gradually involving the remaining clear part of the cornea. By 11.30 o'clock the whole cornea had become opaque, but a few bubbles could still

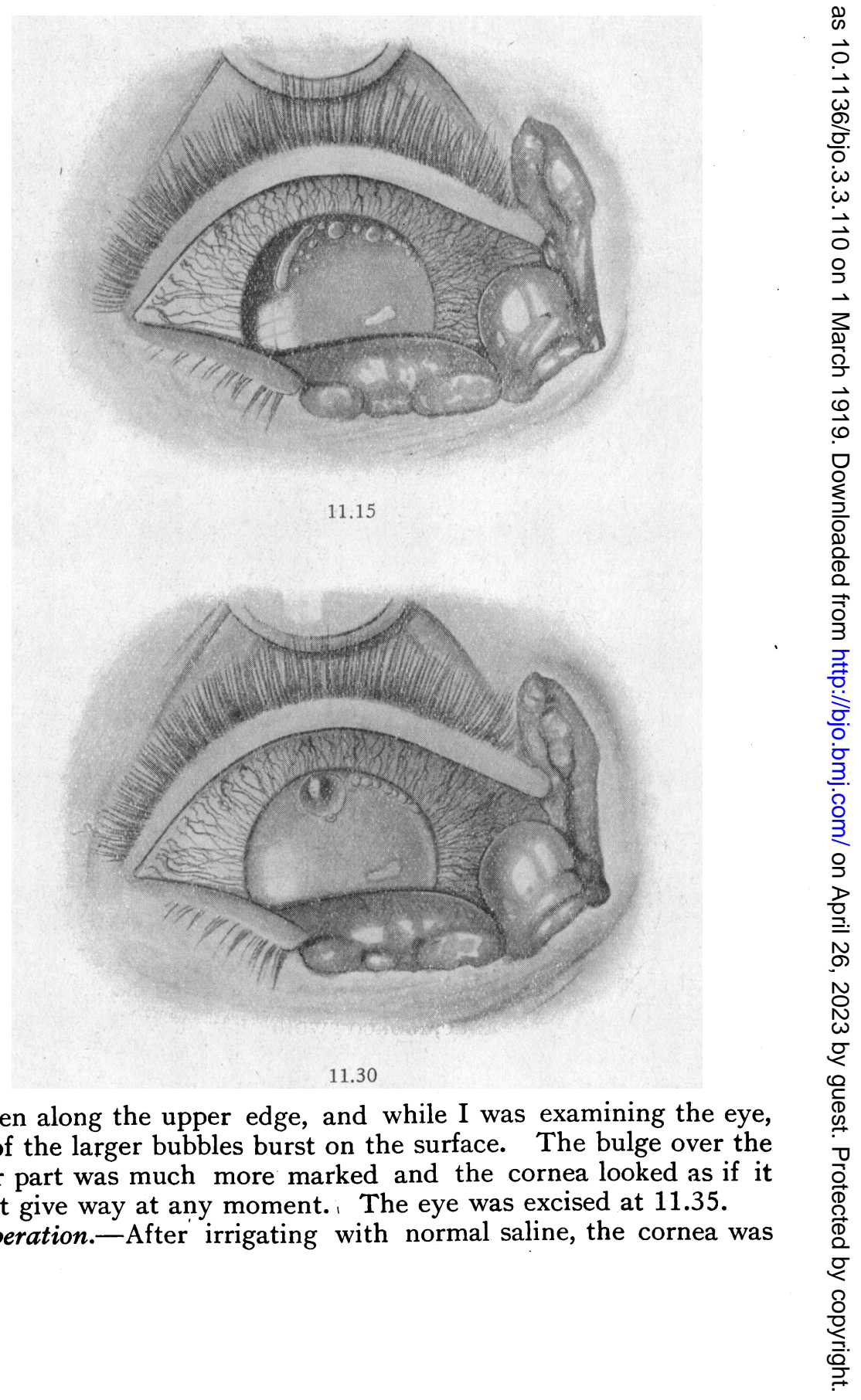

be seen along the upper edge, and while I was examining the eye, one of the larger bubbles burst on the surface. The bulge over the lower part was much more marked and the cornea looked as if it might give way at any moment.i The eye was excised at 11.35 .

Oberation.-After irrigating with normal saline, the cornea was 
removed and the contents of the globe were carefully eviscerated. Except for a small mass of exudate which was found lying on the ciliary body to the outer side, the contents of the globe seemed healthy. The interior of the sclera was thoroughly irrigated with normal saline and the sclera then removed, a small collar being left round the nerve head. The socket was then swabbed out thoroughly with hydrogen peroxide, irrigated with normal saline, and a few drops of 15 per cent. argyrol were left in. A pad and bandage were applied for an hour, and the socket.was then left uncovered. Irrigations with weak hydrogen peroxide were ordered three hourly, and a few drops of 15 per cent. argyrol were applied night and morning.

Next day the socket looked clean and there was no sign of gas, but the upper lid was rather swollen and red, and the conjunctiva still chemosed. The patient's general condition was excellent and he had no pain. On the following morning, as the upper lid was distinctly more swollen, three hourly fomentations were ordered and warm boric lotion was substituted for the hydrogen peroxide. In two days the swelling had disappeared and the socket was quite clean and healthy. On May 19 the patient was transferred to England.

The temperature chart showed no special point of interest, the temperature remaining practically normal from May 6 to May 19 , except for a rise to $100^{\circ} 6^{\circ} \mathrm{F}$. on the morning after the operation.

\section{Laboratory report by Major S. W. Patterson, R.A.M.C.}

Captain Pringle's eye specimen for gas organisms.

In situ the blurred cornea of the eye showed small gas bubbles as described above. After irrigating with saline solution, a glass capillary pipette was pushed into the corneal substance and a small amount of fluid obtained. Films of this fluid, when stained, showed no organisms, but cultures gave both streptococci and staphylococci. A small portion of gassy cornea was snipped off and dropped into a mince meat tube. After incubation, a growth was noted of streptococci, and staphylococci, diphtheroids, and numerous Gram-positive rods of varying lengths; there were a few bubbles of gas formed, and the meat was slowly digested; subculture after heating to $80^{\circ} \mathrm{C}$. for 15 minutes showed subterminally sporing Gram-positive rods.

The purulent iris on culture gave streptococci and staphylococci; and cultures from the vitreous showed the same micro-organisms.

In sections, the cornea showed separation of the fibres by vacuoles, in the walls of which the corneal cell nuclei were proliferating; while at the surface and periphery of the cornea, the vacuoles also contained polymorph cells which had wandered in.

The sclera showed considerable engorgement, and in places extravasation of blood had occurred. 
The micro-organisms found are, therefore, those usually met with in infected war wounds; i.e., streptococci and staphylococci, B. perfringens, and B. sporogenes. There were also a few forms suggestive of vibrion septique, but these last were not isolated in pure culture.

\section{Remarks}

One of the most striking features in these three cases was the rapid involvement of the cornea. In each it was only a matter of a few hours, while in the last case the upper third of the cornea became infiltrated in 45 minutes. I do not know of any other condition in which this occurs.

I was exceptionally fortunate in being able to observe the actual formation of the gas bubbles in the corneal substance in the last case. This, doubtless, occurred in the other two cases as well, but in them the condition had advanced so far that the bubbles were either hidden by the opaque cornea or had burst on the surface.

It would appear as though it were necessary to have some break in the corneal epithelium, and possibly in Bowman's membrane, before the gas organisms can gain access to the corneal substance. In my first two cases this break was caused by actual wounds of the cornea, and in the last case by an ulcer following exposure of the cornea. When wounds of the cornea occur, it is probable that the infection is carried in by the foreign body, but cases where an ulcer is the starting point of infection are more difficult to explain. My last case would lead one to believe that here the wounds of the . soft parts round the eye are the seat of the primary infection, and that the organisms are carried into the conjunctival sac in the discharge from these wounds. Why the soft parts themselves do not become "gassy" is probably due to their excellent blood supply, as mentioned in the early part of this article.

It is unfortunate from the observer's point of view that in each case the eye was removed while the cornea alone was involved, as it would have been extremely interesting to note the spread of the infection. Major Patterson's report shows that only streptococci and staphylococci were isolated from the contents of the globe and that beyond inflammatory reaction the sclera showed no signs of actual gas infection. It is probable that the sclera would offer considerably more resistance to the development of gas organisms than the damaged cornea, which would explain the fact that I was able to remove the eye before this structure was involved. As streptococci and staphylococci were isolated from the iris and vitreous, infection had evidently spread through Descemet's membrane, which had become disorganized, into the interior of the globe, and whether or not the blood supply to the iris and ciliary body had anything to do with the barrier offered to the inroads of 
the gas organisms in this direction, one can only conjecture. It was impossible at the time of the operation to obtain a specimen from the aqueous, as the cornea gave way as soon as the globe was touched.

The three drawings of the eye have been done for me by Sergt. Maxwell, R.A.M.C., from rough sketches made at the time, and I should like to take this opportunity of thanking him for the excellent way in which he has carried out the work. They give a very good idea of the appearance of the cornea at different stages, and also show the entrance wound at the inner canthus, and the masses of chemosed conjunctiva filling up the gap in the lower lid. In the first drawing the large area of opaque cornea is clearly seen, with the ulcer down and in, and the rows of bubbles slightly ahead of the advancing infiltration. The second drawing gives the appearance half an hour later, when the bubbles had run together, and the infiltration had spread further towards the upper edge of the cornea; whilst the last drawing shows the cornea completely opaque, with the large bubble at the upper edge, which burst as the sketch was being made.

\title{
A NEW TEST TYPE FOR THE DETECTION OF MALINGERERS IN ARMY WORK
}

\author{
BY \\ Alan W. Sichel, B.A., M.B., Ch.B. (Edin.) \\ CAPTAIN, R.A.M.C.
}

IN examining the visual acuity of men in the Army the ophthalmic surgeon has to contend with malingering, and in many cases the detection of a malingerer is a matter of some difficulty.

Out of 1,956 cases where the visual acuity was examined at No. - Ophthalmic Centre during a period of twelve months, 101 cases of malingering were recorded, representing about 5 per cent. of all eye cases seen.

By malingering in this connection is meant the refusal of men to admit having as high a visual acuity as they really possess, whether they be emmetropic or have an error of refraction small or moderate in amount.

Malingering in the Army differs from that met with by the oculist in civil practice in this respect; in the latter case the malingerer usually maintains that he is blind in one or both eyes, or at any rate that he has no useful vision in one or both eyes in order to claim compensation for an injury alleged to have been caused by his work. In Army work, on the other hand, the type of 